$C=0, m=0, N=0$, and $\hat{N}=n_{p_{1}}+\cdots+n_{p_{i}}$. Thus, the composite statistics of the training class are

$$
\begin{aligned}
\hat{\mu}= & \sum_{i=1}^{l}\left(\frac{n_{p_{i}}}{\hat{N}}\right) \mu_{p_{i}} \\
\hat{C}= & \sum_{i=1}^{l}\left(\frac{n_{p_{i}}-1}{\hat{N}-1}\right) C_{p_{i}}+\sum_{i=1}^{l}\left(\frac{n_{p_{i}}}{\hat{N}-1}\right) \mu_{p_{i}} \mu_{p_{i}}{ }^{T} \\
& -\left(\frac{\hat{N}}{\hat{N}-1}\right) \hat{\mu} \hat{\mu}^{T} .
\end{aligned}
$$

\section{A Computationally Simple Procedure for Imagery Data Compression by the Karhunen-Loève Method}

\section{K. SHANMUGAM AND R. M. HARALICK}

\begin{abstract}
Of the several methods that have been proposed for imagery data compression, the Karhunen-Loève procedure minimizes the meansquare error between the original and reconstructed imagery data. In spite of its optimality property, the Karhunen-Loève procedure has not been widely used because of its computational complexity. The main difficulty is in the computation of the eigenvectors and the eigenvalues of the covariance matrix of the imagery data since the dimension of the covariance matrix is usually large.

A computationally short procedure for calculating the eigenvalues and eigenvectors of the covariance matrix is presented. We show that the eigenvalues and eigenvectors of the $N \times N$ bisymmetric covariance matrix can be obtained from the eigenvalues and eigenvectors of two $N / 2 \times N / 2$ submatrices. Since the eigenvector calculations are proportional to the third power of the matrix dimension, the proposed procedure reduces the computations by a factor of four.
\end{abstract}

\section{INTRODUCTION}

Imagery data in general contain a large amount of redundant information because of the high positive correlation between the gray levels of spatially adjacent image elements. Several imagery data compression techniques have been proposed recently for removing this redundant information [1]-[4]. Of these methods, the principal component method (based on the KarhunenLoeve expansion) minimizes the mean-square error between the original and compressed imagery data.

In the principal components method, the image is first split into a number of small mutually exclusively spatial regions or windows, and the gray levels of these regions are treated as $N$-dimensional vectors. (These vectors are assumed to have a mean of zero; if not, the mean vector can be calculated and subtracted from each of these vectors.) The image is then a collection of these vectors. These $N$-dimensional vectors $X_{1}, X_{2}$, $\cdots, X_{k}$ are then projected into some smaller $r$-dimensional subspace having maximal variance. In this way the $N$ components of the original data may be expressed in terms of $r$ components, thus achieving a data compression of $N / r$.

An optimal basis for the $r$-dimensional subspace is the set of $r$ eigenvectors $V_{1}, V_{2}, \cdots, V_{r}$ corresponding to the $r$ largest eigenvalues of the sample covariance matrix of $X_{1}, X_{2}, \cdots, X_{k}$. The reconstructed value of the imagery data in the $j$ th subimage

Manuscript received April 25, 1972; revised August 7, 1972.

The authors are with the Center for Research and the Department of Electrical Engineering, University of Kansas, Lawrence, Kans. 66044. region (window) is given by

$$
X_{J}^{*}=\sum_{i=1}^{r}\left[V_{l}^{\prime} \cdot X_{J}\right] V_{l}
$$

where $V_{l}^{\prime}$ denotes the transpose of $V_{t}$. The principal component procedure minimizes the mean-square error

$$
E=\frac{1}{k} \sum_{i=1}^{k}\left\|X_{i}-X_{i}^{*}\right\|^{2}
$$

The minimum value of $E$ for $r$ projections is given by

$$
E_{r}^{*}=\sum_{i=r+1}^{N} \lambda_{t}
$$

where $\lambda_{t}$ are the $N-r$ smallest eigenvalues of the covariance matrix.

In spite of its optimality, the principal component method has not been widely used because of its computational complexity [5]. The main difficulty is in the computation of the eigenvalues and the eigenvectors of the $N \times N$ covariance matrix. The window sizes for large images range from $4 \times 4$ to $10 \times 10$, leading to $16 \times 16$ to $100 \times 100$ covariance matrices. The calculations for the eigenvalues and eigenvectors of these large-sized matrices require a considerable amount of computation time and storage.

We are presenting in this paper a computationally short procedure for calculating the eigenvalues and eigenvectors of the covariance matrix. This simplification results from the bisymmetric properties of the covariance matrix, which is an outgrowth of using a square window as a sampling device over an image. We show that the eigenvalues and eigenvectors of the $N \times N$ bisymmetric covariance matrix can be obtained from the eigenvalues and eigenvectors of two $N / 2 \times N / 2$ submatrices. Since the eigenvector calculations are proportional to the third power of the matrix dimension, the proposed procedure reduces the computations by a factor of four.

\section{Construction of the Sample Covariance Matrix}

The entries in the covariance matrix of a given image are obtained by calculating the average covariance of all the elements in the image that has the same spatial relationship as the entry being considered. This procedure can best be illustrated using an example.

The $4 \times 4$ array shown in Fig. 1(a) represents a small image whose data are to be compressed. The image elements are labeled from 1 to 16. The size of the window for this example is $2 \times 2$ and the arrangement of components of the data vector $X$ within each window is shown in Fig. 1(b). For this image, the average covariance array and the covariance matrix are computed as follows.

Each element in the average covariance array (Fig. 1(c)) is the average covariance of all the elements in the original image, having the same spatial relationship to each other as the element of the covariance array has to the lower center element. For example, the element $c$ in the covariance array is located along the $45^{\circ}$ diagonal line from the lower center (reference) element $e$. Accordingly, the entry $c$ is the average covariance of 9 pairs of similarly spatially related image elements $(5,2),(6,3),(7,4),(9,6)$, $(10,7),(11,8),(13,10),(14,11)$, and $(15,12)$. Other elements of the average covariance array are calculated using similar spatial relationships. The entries in the $4 \times 4$ covariance matrix (Fig. 1(d)) for the image can be obtained from the data contained in the average covariance array. For example, the entry at the second row, third column of the covariance matrix represents the covariance of $x_{2}$ and $x_{3}$. Elements $x_{3}$ and $x_{2}$ are along the $45^{\circ}$ diagonal of the window and the element $c$ in the average 


\begin{tabular}{|c|c|c|c|}
\hline 1 & 2 & 3 & 4 \\
\hline 5 & 6 & 7 & 8 \\
\hline 9 & 10 & 11 & 12 \\
\hline 13 & 14 & 15 & 16 \\
\hline
\end{tabular}

(a)

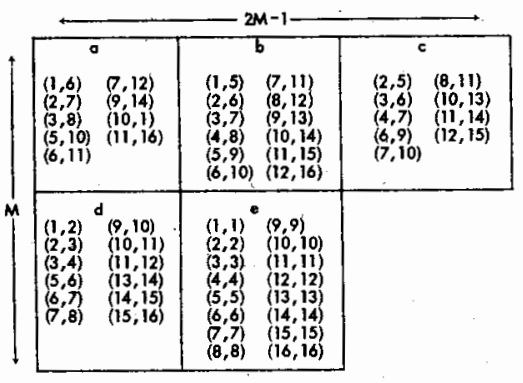

(c)

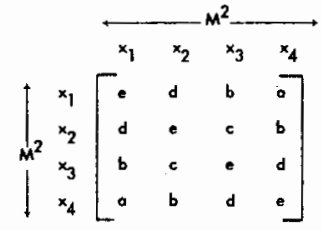

(d)
Fig. 1. (a) Original image. (b) Arrangement of variables within window. (c) Average covariance array. (d) Covariance matrix.

\begin{tabular}{|l|l|l|l|}
\hline$x_{1}$ & $x_{2}$ & $x_{3}$ & $x_{4}$ \\
\hline$x_{5}$ & $x_{6}$ & $x_{7}$ & $x_{8}$ \\
\hline$x_{9}$ & $x_{10}$ & $x_{11}$ & $x_{12}$ \\
\hline$x_{13}$ & $x_{14}$ & $x_{15}$ & $x_{16}$ \\
\hline
\end{tabular}

(a)

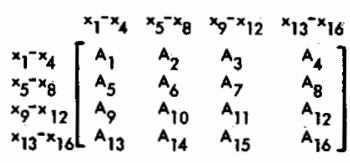

(b)
Fig. 2. (a) Elements of $X$ in $4 \times 4$ window. (b) Partitioned form of covariance matrix $C$ of $X$.

covariance array bears the same relationship to the lower center (reference) element $e$. Hence, the entry $c$ is used to fill in the $(2,3)$ element in the covariance matrix. Similarly, the remaining entries in the covariance matrix are obtained.

The procedure described in the preceding paragraphs can be used for square windows of any size $M \times M$, with $M$ less than the overall dimension of the image itself. In the following we will restrict our attention to windows of size $M \times M$, where $M$ is even. The sample covariance matrix obtained from the average covariance array has a bisymmetric ${ }^{1}$ form. Also, the $M^{2} \times M^{2}$ covariance matrix consists of $\dot{M}$ submatrices of dimension $M \times M$. These submatrices appear in a bisymmetric form within the covariance matrix. Let us consider the following example to further illustrate the bisymmetric properties of $C$.

Fig. 2(a) shows the arrangement of the components of $X$ within a $4 \times 4$ window, and the $16 \times 16$ covariance matrix of $X$ is shown in a partitioned form in Fig. 2(b). The $4 \times 4$ submatrices $A_{l}$ represent the covariance of the elements of one row of the window with the elements of another row of the window. The matrices $A_{1}, A_{6}, A_{11}$, and $A_{16}$ represent the covariance of the elements of a row with the elements of the same row. The spatial relationships existing between the elements of row 1 is the same as the spatial relationships between the elements of row 2 , row 3 , or row 4 . Since the entries in all these matrices are obtained from the average covariance array using the spatial relationship between the elements, $A_{1}, A_{6}, A_{11}$, and $A_{16}$ are identical. Next, let us consider the matrices $A_{2}, A_{7}, A_{12}, A_{5}, A_{10}$, and $A_{15}$, which represent the covariance between the element of

\footnotetext{
${ }^{1}$ Bisymmetric: An $N \times N$ matrix $A$ is bisymmetric if and only if

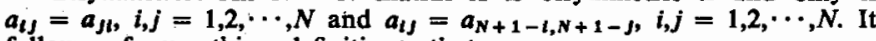
follows from this definition- that $a_{l j}=a_{\mu}=a_{N+1-i, N+1-j}=$ $a_{N+1-j, N+1-t, i, j=1,2, \cdots, N}$
}

one row of the window and the elements of an adjacent window. For instance, $A_{2}$ represents the covariance between the elements $x_{1}, x_{2}, x_{3}, x_{4}$ and $x_{5}, x_{6}, x_{7}, x_{8}$. Referring to Fig. 2(a), the spatial relationships between $x_{1}, x_{2}, x_{3}, x_{4}$ and $x_{5}, x_{6}, x_{7}, x_{8}$ and the spatial relationships between $x_{5}, x_{6}, x_{7}, x_{8}$ and $x_{9}, x_{10}, x_{11}, x_{12}$ are the same. Hence, the entries in $A_{2}$ and $A_{7}$ will be the same. Extending this reasoning, it is easy to see that $A_{2}, A_{7}, A_{12}, A_{5}, A_{10}$, and $A_{15}$ are identical. Similarly, it can be shown that $A_{3}, A_{8}, A_{9}$, and $A_{14}$ are the same and that $A_{4}$ and $A_{13}$ are also identical. Hence, the form of the covariance matrix becomes

$$
C=\left[\begin{array}{cc:cc}
A_{1}, & A_{2} & A_{3} & A_{4} \\
A_{2}^{\prime} & A_{1} & A_{2} & A_{3} \\
\hdashline A_{3}^{\prime} & A_{2}^{\prime} & A_{1}, & A_{2} \\
A_{4}^{\prime} & A_{3}^{\prime} & A_{2}^{\prime} & A_{1}
\end{array}\right]
$$

where $A_{i}^{\prime}$ denotes the transpose of $A_{i}$. Thus, the bisymmetric property of the covariance matrix is inherent in the window image sampling process. This example also illustrates that the $M^{2} \times M^{2}$ covariance matrix consists of $M$ submatrices of dimension $M \times M$ arranged in a bisymmetric form. The submatrices are not symmetric; however, for each matrix, $a_{i j}=$ $a_{M+1-t, M+1-\jmath}$.

The symmetry properties of the covariance matrix lead to a computationally simple procedure for the eigenvalue-eigenvector calculations. The procedure we will develop in the next section is similar to a method given by Ray and Driver [6] for decomposition of the Karhunen-Loève series representation of stationary random process.

\section{EIgENVALUES AND EIgENVECTORS OF THE Covariance Matrix}

We now show that the eigenvalues and eigenvectors of the $2 m \times 2 m$ covariance matrix $C$ can be obtained by calculating the eigenvalues and eigenvectors of two $m \times m$ submatrices of $C$. This simplification results from the bisymmetry properties of $C$ and the simplified procedure is developed through lemmas 1-3.

\section{Lemma 1}

The covariance matrix $C$, of dimension $2 m \times 2 m$, can be partitioned into $m \times m$ submatrices of the following form:

$$
C=\left[\begin{array}{c:c}
\boldsymbol{A} & \boldsymbol{B P} \\
\hdashline \boldsymbol{P B} & \boldsymbol{A}
\end{array}\right]
$$

where $A$ and $B$ are $m \times m$ submatrices of $C$, and $P$ is an $m \times m$ matrix with ones along the NE-SW diagonal and zeros elsewhere; i.e., the $(i, j)$ th element of $P$ is given by

$$
(P)_{i j}= \begin{cases}1, & \text { for } j=m+1-i \\ 0, & \text { otherwise. }\end{cases}
$$

The proof of lemma 1 follows from the construction of $C$.

\section{Lemma 2}

The eigenvectors $V_{l}, i=1, \cdots, 2 m$, of $C$ have either one of the following two forms:

$$
V_{i}=\left[\begin{array}{c}
v_{i} \\
P v_{i}
\end{array}\right] \quad \text { or } \quad V_{i}=\left[\begin{array}{c}
v_{i} \\
-P v_{i}
\end{array}\right]
$$

where $v_{i}$ is an $m \times 1$ column vector.

Proof: The characteristic equation of $C$ is given by $C Y=$ $\lambda Y$, where $Y$ is an eigenvector of $C$ corresponding to the eigenvalue $\lambda$. We want to prove that the $i$ th component of $Y$, denoted $y_{i}$, satisfies

$$
y_{t}= \pm y_{2 m+1-t}
$$


We begin by writing the characteristic equation in the form

$$
\lambda y_{i}=\sum_{j=1}^{2 m} c_{i j} y_{j}, \quad i=1,2, \cdots, 2 m
$$

or

$$
\lambda y_{2 m+1-i}=\sum_{j=1}^{2 m} c_{2 m+1-i, j} y_{j}
$$

where $c_{i j}$ is the $(i, j)$ th element of $C$. We may also write $(7)$ as

$$
\lambda y_{2 m+1-i}=\sum_{j=1}^{2 m} c_{2 m+1-t, 2 m+1-j} y_{2 m+1-j}, \quad i=1,2, \cdots, 2 m \text {. }
$$

The bisymmetric property of $\dot{C}$ yields $c_{2 m+1-t, 2 m+1-\jmath}=c_{i \jmath}$, and hence we may write the preceding equation as

$$
\lambda y_{2 m+1-1}=\sum_{j=1}^{2 m} c_{i, j} y_{2 m+1-j}
$$

Equations (6) and (8) both represent $2 m$ equations in $2 m$ unknowns. Letting $y_{2 m+1-i}=Z_{i}$, (8) becomes

$$
\lambda Z_{i}=\sum_{j=1}^{2 m} c_{i j} Z_{j}
$$

From (6) and (9) it is obvious that the solution for the $y_{t}$ is also the solution for the $Z_{i}$, i.e., $y_{t}$ and $y_{2 m+1-i}$ have the same solution.

Since the signs of the eigenvectors are not unique, forcing the norm of the eigenvectors to 1 makes $y_{i}= \pm y_{2 m+1-i}, i=$ $1, \cdots, 2 m$. Thus we establish (4), and hence the proof of the lemma.

\section{Lemma 3}

The $2 m$ eigenvalues $\lambda_{1}, \lambda_{2}, \cdots, \lambda_{2 m}$ and the corresponding eigenvectors $V_{1}, V_{2}, \cdots, V_{2 m}$ of $C$ divide into the following two groups: part I,

$$
\lambda_{i}=\lambda_{i}^{+} \quad V_{i}=\left[\begin{array}{c}
\dot{v}_{i}^{+} \\
P v_{i}^{+}
\end{array}\right], \quad i=1,2, \cdots, m
$$

and part II,

$$
\lambda_{i}=\lambda_{i}^{-} \quad V_{i+m}=\left[\begin{array}{c}
v_{i}^{-} \\
-P v_{t}^{-}
\end{array}\right], \quad i=1,2, \cdots, m
$$

where $\lambda_{i}{ }^{+}$and $v_{i}{ }^{+}$are the eigenvalues and eigenvectors ${ }^{2}$ of the $m \times m$ submatrix $A+B$ and $\lambda_{i}{ }^{-}$and $v_{i}^{-}$are the eigenvalues and eigenvectors of the $m \times m$ submatrix $A-B$; i.e.,

$$
\begin{aligned}
& {[A+B] v_{i}^{+}=\lambda_{i}^{+} v_{i}^{+}} \\
& {[A-B] v_{i}^{-}=\lambda_{i}{ }^{-} v_{i}^{-}}
\end{aligned}
$$

where $i=1,2, \cdots, m$.

Proof: The characteristic equation of $C$ is

$$
C V_{i}=\lambda_{i} V_{t}
$$

Using the partitioned form of $C$, we may write the preceding equation as

$$
\left[\begin{array}{cc}
A & B P \\
P B & A
\end{array}\right] V_{i}=\lambda_{i} V_{i}
$$

Lemma 2 gives two forms of $V_{i}$, and substituting the first form given in (4), we can write (14) as

$$
\left[\begin{array}{cc}
A & B P \\
P B & A
\end{array}\right]\left[\begin{array}{c}
v_{i} \\
P v_{i}
\end{array}\right]=\lambda_{i}\left[\begin{array}{c}
v_{i} \\
P v_{i}
\end{array}\right]
$$

2 The vectors $v_{t}^{+}$and $v_{t}^{-}$are normalized to give $\left\|v_{t}{ }^{+}\right\|^{2}=\left\|v_{t}-\right\|^{2}=\frac{1}{2}$ so that $\left\|V_{1}\right\|^{2}=1$.
The first $m$ equations of (15) yield

$$
A v_{i}+B P P v_{i}=\lambda_{l} v_{i} \text { or }[A+B] v_{t}=\lambda_{i} v_{t}
$$

since $P P=I$.

Comparing (16) with the characteristic equation of the matrix $A+B$ given in (12), we see that $\lambda_{i}=\lambda_{i}{ }^{+}$and $v_{i}=v_{i}{ }^{+}$; and hence the proof of the first part of the lemma. Similarly, by taking the second form of $V_{l}$ given in (4) we can prove part II of lemma 3. This completes the proof of lemma 3, which shows that the eigenvalues and eigenvectors of the $2 m \times 2 m$ covariance matrix $C$ can be obtained from the eigenvalues and eigenvectors of two $m \times m$ submatrices $A+B$ and $A-B$.

\section{Conclusions}

We have presented a procedure which simplifies the computational complexity involved in calculating the eigenvectors and eigenvalues of the covariance matrix of imagery data. The procedure is based on the decomposition of the covariance matrix $C$ as

$$
C=\left[\begin{array}{c:c}
\boldsymbol{A} & \boldsymbol{B P} \\
\hdashline \boldsymbol{P B} & \boldsymbol{A}
\end{array}\right] .
$$

We have shown that the eigenvalues and eigenvectors of the $2 m \times 2 m$ covariance matrix can be obtained from the eigenvalues and eigenvectors of the $m \times m$ submatrices $A+B$ and $A-B$. Since the eigenvector calculations are proportional to the third power of the dimension of the matrix, the proposed procedure reduces the computations by a factor of four.

Also, the eigenvectors of the covariance matrix has to be stored or transmitted to the receiver for reconstructing the imagery data according to (1). The symmetry property of the eigenvectors given in (10) and (11) enables us to store or transmit only half of the components of the eigenvectors. This results in a considerable saving in transmission time, especially if the dimension of the eigenvector is large.

\section{REFERENCES}

[1] Special Issue on Digital Picture Processing, Proc. IEEE, vol. 60, pp. 768809 , July 1972.

[2] W. K. Pratt, J. Kane, and H. C. Andrews, "Hadamard transform image coding," Proc. IEEE, vol. 57, pp. 58-68, Jan. 1969.

[3] M. Tasto and P. A Wintz, "Image coding by adoptive block quantization," IEEE Trans. Commun. Technol., vol. COM-19, pp. 957-972, Dec. 1971.

[4] R. M. Haralick, J. Young, D. Goel, and K. Shanmugam, "A comparative study of transform data compression techniques for digital image transmission," presented at the 1972 Nat. Electron. Conf., Chicago, Ill., Oct. 9-11, 1972.

[5] C. A. Andrews, J: M. Davies, and G. R. Schwarz, "Adaptive data compression," Proc. IEEE, vol. 55, pp. 267-277, Mar. 1967.

[6] W.D. Ray and R. M. Driver, "Further decomposition of the KarhunenLoève series representation of stationary random process," IEEE Trans. Inform. Theory, vol. IT-16, pp. 663-668, Nov. 1970.

\section{On User Supplied Evaluations of Time-Shared Computer Systems}

\author{
JERROLD M. GROCHOW
}

\begin{abstract}
Comments are made regarding the collection of user preference data for varying characteristics of time-sharing systems. These "utility functions," when determined for a number of variables, can be used as an aid to managers and designers of time-sharing service facilities.
\end{abstract}

Manuscript received February 17, 1972; revised October 23, 1972. This work was supported by a grant from the Office of Information Processing Services, Massachusetts Institute of Technology.

The author was with the Sloan School of Management, Massachusetts
Thices, Massachusetts Institute of Technology. Institute of Technology, Cambridge, Mass. 02139. He is now with American Management Systems, Arlington, Va. 22209. 